\title{
Eine Untersuchung über die possessiven Konstruktionen im Udmurtischen und ihre Entwicklung
}

Светлана Едыгарова: Категория посессивности в удмуртском языке [Die Kategorie der Possessivität im Udmurtischen]. Dissertationes Philologiae Uralicae Universitatis Tartuensis 7. Tartu 2010. $289 \mathrm{~S}$.

Edygarovas Dissertation befasst sich mit udmurtischen Konstruktionen, die Possessivität zum Ausdruck bringen, sowie mit solchen, in denen ein Px. verwendet wird, obwohl sie nicht unbedingt Possessivität im eigentlichen Sinn ausdrücken. Im Vordergrund stehen die Verwendung dieser Konstruktionen und ihre Relation zu semantischen Gegebenheiten, d. h. die Frage, warum und wann die jeweilige Konstruktion verwendet wird $($ S. 8, 67). Die Verf. untersucht eingehend auch die Morphologie der Px., u. a. die Frage, welche seman- tische Motivation hinter der lautlichen Variation eines Suffixes steht. Ferner wird die synchrone und diachrone Untersuchung der Px. der uralischen Sprachen ausführlich referiert (S. 30-40). Verdienstvoll beleuchtet Edygarova die Geschichte der possessiven Konstruktionen während der Sonderentwicklung des Udmurtischen, indem sie u. a. das Udmurtische mit seinen nächstverwandten Sprachen, dem Komisyrjänischen und Komipermjakischen, und Belege aus frühen udmurtischen Texten mit entsprechenden Beispielen aus der heutigen Sprache vergleicht (S. 158-16o, 178-179). Die grundlegende Gliederung der possessiven Konstruktionen, die den Ausgangspunkt der vorliegenden Arbeit bildet, wird zweimal in komprimierter Form (S. 67, 256-257) sowie ausführlicher in Kapitel 2 dargestellt. Die Belege 
werden so geschrieben, wie sie in den Quellen begegnen. Aus theoretischen Untersuchungen übernommene Belege werden in lateinischer Transkription wiedergegeben. Sowohl hinsichtlich der Orthografie, der Glossierung wie auch der Sprache ist die Dissertation im Wesentlichen überaus sorgfältig abgefasst. Die Verf. beherrscht die Konventionen und die Terminologie russischsprachiger sprachwissenschaftlicher Abhandlungen. An einigen Stellen wurde ein Terminus versehentlich mit einem anderen, mitunter sogar gegensätzlichen Begriff verwechselt: z. В. вторичное согласование pro вторичное склонение (S. 136) und обладаемыци pro обдалатель (S. 35 u.). Solche Lapsus sind insofern bedauerlich, als sie Leser, die mit dem behandelten Thema oder der russischen Terminologie nicht gründlich vertraut sind, in Verwirrung bringen können.

\section{Die possessive Relation}

Edygarovas zweiter Ausgangspunkt neben den Konstruktionen ist die Semantik. Die possessive Relation ist die Beziehung zwischen zwei Designaten. Freilich ist nicht jede Relation possessiv. Eine Voraussetzung ist z. B., dass die Designate in einer Art hierarchischem Verhältnis zueinander stehen, so dass erkennbar ist, dass das eine dem anderen gehört. Eine natürliche possessive Beziehung ist z. B. der Autoschlüssel oder „Schlüssel des Autos", während das Auto des Schlüssels weniger wahrscheinlich ist. Die Relation wird also u. a. von der Semantik des Besitzes und des Besitzers stark beeinflusst. Die als possessiv aufgefassten Relationstypen sind in verschiedenen Sprachen weitgehend gleich, während die Art der sprachlichen Umsetzung dieser Relationen eher sprachspezifisch ist. Der universale semantische Kern der Possessivität umfasst z. B. nach dem von Edygarova zitierten Bernd Heine folgende Untertypen: 1. physische (momentane) Possessivität: Mein Glas ist leer. 2. zeitliche (okkasionelle) Possessivität: Ich habe ein Auto, mit dem ich zur Arbeit fahre (aber es gehört meiner Frau). 3. bleibende (inhärente) Possessivität: Mein Auto geriet in einen Unfall. 4. unveräußerliche Possessivität (Körperteile, Verwandte): Ich habe blaue Augen / zwei Schwestern. Mein Vater, meine Augen. 5. abstrakte Possessivität: mein Schnupfen, meine Gedanken. I have a missing tooth. 6. unbelebte unveräußerliche Possessivität (Teil-Gesamtheit): Dieser Baum hat einige Zweige. Die Decke dieses Zimmers. 7. unbelebte veräußerliche Possessivität (Besitz vom Besitzer trennbar): Die Stühle dieses Zimmers. That tree has crows on it. 
Auf dieser Grundlage gelangt Edygarova zu der kühnen Entscheidung, auch Konstruktionen in die Betrachtung einzubeziehen, die unbestreitbar possessive Relationen zum Ausdruck bringen, in der traditionellen Grammatik des Udmurtischen jedoch nicht in Verbindung mit der attributiven Possessivität erwähnt werden (S. 67-68). Diese stellen tatsächlich den größten Teil der neun Konstruktionen. Bei diesen von Edygarova als semantisch possessiv klassifizierten Konstruktionen handelt es sich um solche, bei denen der Besitzer im Elativ steht, der Besitzer mit dem Ableitungssuffix -ala / -lo markiert wird, der Besitz vor dem Bezugswort mit dem Ableitungssuffix - o oder dem Instrumental -en, der Besitz (oder genauer gesagt, sein Fehlen) mit dem Karitativsuffix -tem oder in Verbkonstruktionen mit dem Abessiv -tek bezeichnet wird. Neben diesen stehen im Mittelpunkt der Betrachtung natürlich Konstruktionen, die von jeher als possessiv klassifiziert und in Zusammenhang mit der Verwendung der Possessivsuffixe erwähnt wurden. Im Udmurtischen sind alle vier Grundtypen der attributiven Besitzkonstruktion in Gebrauch (S. 24, 33). Edygarova berücksichtigt sowohl die Terminologie der türkischen Grammatiktradition, in der man von izafet-Konstruktionen spricht und die auf das Udmurtische angewandt wurde, als auch die entsprechenden Termini der westlichen Tradition: head-marking, dependant-marking, double-marking und no-marking: (a) double-marking (izafet Typ I): ataj-len jurt-ez (VaterGEN Haus-3SG) 'das Haus des Vaters' (b) dependant-marking (izafet Typ II): Kola-len milkid (Kol'a-GeN Gemüt) 'Kol'as Gemüt(sverfassung)'. (c) head-marking (izafet Typ III): mon ki-ja-m (ich-NOM Hand-ILL/ INE-1SG) 'in meiner Hand'. (d) nomarking (izafet Typ IV): korka l'ipet (Haus-Nom Dach-NOM) 'das Dach des Hauses, Hausdach' Edygarova untersucht, welche Konstruktion im Udmurtischen jeweils gewählt wird, und stellt fest, dass dabei die oben erwähnte semantische Qualität der Possessivität eine Rolle spielt. Man kann z. B. eine bleibende Zusammengehörigkeit attributiv mit dem Ableitungssuffix $-o$, eine vorübergehendere dagegen mit dem Instrumental -en ausdrücken: nilpijo kišnomurt 'eine Frau, die Kinder hat', galstuken vorgoron 'ein Mann, der eine Krawatte hat', oder es wird z. B. ein belebter Besitzer mit dem Genitiv gekennzeichnet, während ein unbelebter mit dem Elativ markiert werden kann. Semantische Oppositionen dieser Art veranschaulicht Edygarova mit einer Tabelle in der Zusammenfassung ihrer Untersuchung (S. 229). Zu den Eigenheiten des Udmurtischen zählt u. a., 
dass Bezeichnungen für Körperteile und jüngere Familienmitglieder eine eigene lautliche Variante des Px. erhalten: vgl. śinmiz 'sein Auge' (aber mašinajez 'sein Auto'), suzeriz 'seine kleine Schwester' (aber apajez 'seine große Schwester'). Edygarova betrachtet diese Variation des vokalischen Elements der Possessivsuffixe $(i / i / e)$ auch aus historischer Sicht und kommt zu dem Ergebnis, dass der Wechsel ursprünglich aus phonetischen Gründen entstand, in der heutigen Sprache aber semantische Unterschiede widerspiegelt. Allerdings sind neben der Semantik auch einige phonetische Einschränkungen zu berücksichtigen (S. 29). Bei der Analyse der Unterschiede gelangt Edygarova zu aufschlussreichen Einzelbeobachtungen. Als Beispiel sei erwähnt, dass die Verbindung zwischen dem Mitarbeiter einer Lehranstalt und dieser Anstalt mit einer anderen Konstruktion (Markierung des Besitzers mit dem Elativ) ausgedrückt wird als die des Leiters der Lehranstalt (der Besitzer wird mit dem Genitiv markiert und zusätzlich wird an den Besitz das Px. angefügt) (S. 208). Solche Beobachtungen sind auch für das breite $\mathrm{Pu}$ blikum ausgesprochen wichtig, u. a. für alle, die ein gutes Schriftudmurtisch anstreben.

Auch bei der Betrachtung der Reihenfolge von Kasusendungen und Possessivsuffixen setzt Edy- garova erfolgreich synchrone und diachrone Aspekte ein. Im Udmurtischen ist wie in vielen uralischen Sprachen sowohl die Reihenfolge PxCx als auch CxPx möglich. Edygarova stellt verschiedene bekannte Theorien zur ursprünglichen Reihenfolge und ihrer Entwicklung vor. Sie kommt jedoch zu dem Schluss, dass die Reihenfolge sich heute (und offenbar auch ursprünglich) am jeweiligen Kasus orientiert und von der Semantik abhängt: Wenn der Besitz die Spezifikation erhält, ist die Reihenfolge $\mathrm{PxCx}$ (häufig dann, wenn die Suffixe an einen Besitz mit belebtem Designat angefügt werden, der häufig nur in den grammatischen Kasus auftritt) wie z. B. bei den grammatischen Kasus. Erhält der Besitzer die Spezifikation, ist die Reihenfolge $\mathrm{CxPx}$, wie bei den meisten Lokalkasus (S. 114117). Zusätzlich wechselt in der heutigen Sprache z. B. die Reihenfolge von Adverbialkasus und Px. je nachdem, ob das Px. die grammatische Funktion hat, die nähere Bestimmung des Satzes und das Subjekt zu verbinden (dann ist die Reihenfolge CxPx), oder ob es die Funktion eines possessiven Spezifikators hat, wenn es an das indirekte Objekt tritt (dann ist die Reihenfolge (PxCx) (S. 111).

Bei der Betrachtung der Konstruktionen wählt Edygarova eine weite Perspektive. Mitunter muss 
man die gesamte syntaktische Struktur des Satzes, die Informationsstruktur und sogar satzübergreifende Gesamtheiten berücksichtigen. Edygarova stellt fest, dass in der Temporalkonstruktion ein Px. verwendet wird, wenn sie dasselbe Subjekt hat wie der Hauptsatz, während bei unterschiedlichem Subjekt das bloße Personalpronomen zur Anwendung kommt (S. 152). Da die Verf. auch an anderer Stelle wiederholt auf die Sachlage in den verwandten Sprachen hinweist, hätte sie auch hier anmerken können, dass es z. B. im Finnischen einen entsprechenden Unterschied gibt, der davon abhängt, ob die Referenzbeziehung reflexiv oder nicht reflexiv ist: So ist z. B. in dem Satz Mikko haki autonsa 'Mikko holte sein (eigenes) Auto' der Besitzer das Subjekt des Satzes, während in dem Satz Mikko haki hänen autonsa 'Mikko holte sein Auto (das eines anderen)' der Besitzer (in der Regel, in der Schriftsprache) ein anderer ist als das Subjekt. Die Betonung der Wichtigkeit von Strukturen, Grammatikalisierungserscheinungen, Analogien und Konventionalisierungstendenzen bei der Untersuchung der Possessivität ist eine der Stärken von Edygarovas Abhandlung. Untersuchungen $\mathrm{zu}$ den Possessivsuffixen vernachlässigen häufig diese Bereiche und konzentrieren sich zu sehr auf die Semantik.

\section{Die assoziative Beziehung und andere pragmatische Funktionen}

Im Unterkapitel 3.1.3.1.2. behandelt Edygarova die assoziativen und anderen pragmatischen Funktionen der Possessivsuffixe. In diesem Kapitel geht es auch um die Frage, ob die Possessivsuffixe im Udmurtischen Bestimmtheit zum Ausdruck bringen. Das Ergebnis ist, dass $3 \mathrm{Sg}$. und $2 \mathrm{Sg}$. eher assoziative Relationen als Bestimmtheit ausdrücken. Zusätzlich wird 2 Sg. verwendet, um ein bekanntes und identifiziertes Objekt zu fokussieren, z. B. Sonne, Himmel, Monat, Wetter, Winter. Dies ist typisch für die Volksdichtung, begegnet in der Umgangssprache jedoch seltener. (S. 131, 132, 136.) Die zentrale Schlussfolgerung ist, dass diese Art der Verwendung des Px. im Udmurtischen mit der Verwendung des bestimmten Artikels und der Markierung der Definitheit in vielen Sprachen in einem gewissen Grad, aber nicht völlig übereinstimmt: Im Grunde handelt es sich doch um etwas Anderes.

Die Betrachtung dieser als nicht-possessiv klassifizierten Verwendung der Possessivsuffixe ist nicht das Hauptthema der Abhandlung. Edygarova verzichtet auf den Versuch, z. B. den Unterschied zwischen possessiver und nicht-possessiver Verwendung ex- 
akt $\mathrm{zu}$ definieren. $\mathrm{Zu}$ Beginn weist sie darauf hin, dass nach Ansicht mancher Wissenschaftler die possessive Relation als mentaler Kontakt zwischen zwei Gegenständen oder Sachverhalten aufgefasst werden kann (S. 15), später spricht sie von der nicht-possessiven assoziativen Beziehung (S. 42) und davon, dass es für das Udmurtische typisch ist, die assoziative Beziehung zwischen zwei Gegenständen oder Sachverhalten so $\mathrm{zu}$ markieren, als wäre sie possessiv (S. 63-64), und bezeichnet darüber hinaus die assoziative Beziehung oder uneigentliche Possessivität als einen der semantischen Kerne der Possessivität im Udmurtischen. An anderer Stelle bezeichnet sie jedoch als eigentliche possessive Relationen nur solche, bei denen es sich um ein eigentliches bleibendes Besitzverhältnis handelt (im Wesentlichen der oben angeführte Punkt 3) (S. 15). Dies macht deutlich, dass in der einschlägigen Forschung noch eine ganze Reihe von Definitionsfragen zu klären sind, die auch die Terminologie betreffen. Ihre Belege für Verwendungsweisen des Px., die assoziative Beziehungen bezeichnen, analysiert Edygarova sorgfältig und betont zu Recht die Bedeutung von Hintergrundwissen und Kontextbetrachtung.

\section{Das determinative Suffix}

In der vorliegenden Untersuchung wird im o. a. Kapitel zu einem gewissen Grad auch das sog. determinative Suffix (вьделительно-указательный субфикс) des Udmurtischen behandelt. Mitunter wird es als eine Funktion des Suffixes der 3. Person Singular klassifiziert, mitunter als eigenes Suffix. Zur Klassifizierung bezieht Edygarova nicht Stellug. Sie erwähnt, dass das Suffix EZ im Udmurtischen nach der bereits 1949 veröffentlichten Auffassung von Pozdeeva sogar drei Funktionen hat: es drückt a) Possessivität, b) Bestimmtheit oder Demonstrativität (wörtlich Unterscheidbarkeit) aus oder fungiert c) als Kasusendung des Akkusativs (S. 75). Es ist durchgängig $\mathrm{zu}$ beobachten, dass Edygarova nachgerade pedantisch die gesamte relevante Literatur berücksichtigt, wobei ihr besonderes Augenmerk widersprüchlichen Auffassungen gilt. Hinsichtlich der Quellen geben nur zwei Punkte Anlass zu Kritik. Im Zusammenhang mit dem Determinativsuffix spricht Edygarova einfach nur von Kongruenz, wenn sie auf das Phänomen verweist, bei dem die mit Determinativsuffix versehene Bestimmung und das Bezugswort im selben Kasus stehen (S. 136). Einer der ausführlich begründeten Hauptgedanken in Ala- 
tyrevs Untersuchung (1970) über dieses Suffix besagt jedoch, dass es sich keineswegs um Kongruenz handelt. Edygarova erwähnt, dass vorgebracht wurde, dieses Suffix habe sich auf der Basis der Demonstrativpronomina gebildet und habe demnach keine Verbindung zu den Px., gibt dafür aber keinerlei Quelle an (S. 138). Der Beitrag von Suihkonen über die Funktionen der possessiven und deiktischen Suffixe des Udmurtischen ist Edygarovas Aufmerksamkeit leider entgangen (Suihkonen 2005).

Der „demonstrativ-unterscheidenden Funktion" des Px. der dritten Person (dem sog. Determinativsuffix) sind in der Dissertation nur zwei Seiten gewidmet. Für dieses Phänomen hat Nikolaeva auch die Begriffe Kontrast und Emphase verwendet. Sie spricht von einer selbstständigen identifizierenden Funktion, bei der es keinen Referenten gibt (2003: 140-142). Das "demonstrativ-unterscheidende“ Suffix gilt als typisch vor allem für Adjektive, und nach Ansicht einiger Wissenschaftler hat es in erster Linie substantivierende Funktion (S. 136). Nach V. I. Alatyrevs Ansicht ist die Hauptfunktion des Suffixes demonstrativ, und die unterscheidende Bedeutung ist eine natürliche Folge (1970: 4). Edygarova zufolge verstärkt die Präsenz eines kontrastiven Elements die un- terscheidende Bedeutung, ist aber nicht unentbehrlich. Manchmal ist das kontrastive Element vorhanden, nämlich dann, wenn der Satz mehrere mit Determinativsuffix markierte Elemente enthält, die dann also zueinander in Kontrast stehen. Notwendig sei das kontrastive Element jedoch nicht.

Edygarova begründet nicht näher, weshalb sie das Determinativsuffix im selben Unterkapitel behandelt wie die oben erwähnten assoziativen Beziehungen. Es gibt einige Zwischenformen. Ein Beispiel erinnert sehr an die im Zusammenhang mit dem Determinativsuffix erwähnten Sätze mit kontrastivem Element (hier würden ausnahmsweise die Substantive in Kontrast stehen) (op. cit. 135): śures-a-d val kotma-iz: berdon-ez, šumpoton-ez, danjaśkon-ez (Weg-INE-2SG sein. EX.1PRÄT alle-3SG Weinen-3SG Freude-3Sg Ehre-3sg) 'Auf deinem (Lebens)weg war allerlei: Weinen, Freude, Ehre'. Ein im Komi möglicher Satz, wo man beim Anblick eines Bären ausruft „ošk-izz!“ Bär-3sG, ohne assoziative Beziehung, ist im Udmurtischen nicht möglich. Aber auf einer Wiese kann man ausrufen (S. 135): turin-ez čeber uk! (Gras-3SG schön PART) 'Wie schön das Gras (auf der Wiese) ist'. Edygarovas Beobachtungen bieten wertvolle Anregungen für weiterführende Untersuchungen. Sie stellt zu Recht den 
Gedanken in Frage, das Determinativsuffix habe im Udmurtischen keinen Referenten.

\section{Diachrone Betrachtung}

Edygarova gelangt auch zu interessanten Beobachtungen über die Geschichte der Px. Ihrer Ansicht nach wurden die Px. im Udmurtischen zunächst syntaktisch und erst später possessiv verwendet. Die Bezeichnung der unveräußerlichen Possessivität mit dem Px. ist im Udmurtischen eine junge Erscheinung (S. 158-160). Die Verwendung des Ablativs anstelle des Genitivs beim Objekt im heutigen Udmurtischen ist ausgesprochen spät aufgekommen, wie ältere Texte zeigen. Früher hatte der Kasuswechsel von Ablativ und Genitiv eine auf der Semantik des Verbs beruhende Motivation. (S. 178-179, 182). Edygarova geht auch kurz auf das Perfekt ein, bei dem im Udmurtischen Possessivsuffixe verwendet werden. Dies wiederum geht offensichtlich zumindest teilweise auf türkischen Einfluss zurück. Auch Vergleiche mit dem Komi zeigen, dass während der Sonderentwicklung des Udmurtischen im Ausdruck der Possessivität und in der Verwendung der Px. viele Veränderungen eingetreten sind. Eine genauere Untersuchung dieser Entwicklung wäre ebenfalls ein Thema für die weitere Forschung. Auch lautliche Veränderungen sind spät eingetreten. Edygarova vermutet den Grund dafür, dass $e$ in einigen mit Pronomina verbundenen Px. zu $i$ wurde (s. B. soje er-1SG > soji), in dem Bestreben, Homonymie mit der Akkusativform zu vermeiden (z. B. soje 'ihn') (S. 86). Diese Erklärung erscheint nicht glaubhaft, denn die betr. Formen haben eine unterschiedliche Satzstellung, die Missverständnisse ausschließt. Zum anderen erhebt sich die Frage, warum z. B. die Homonymie der Suffixe des Illativs und der 1 SG keine Lautveränderungen verursacht hat (z. B. mašinaje 'ins Auto, mein Auto'). Edygarovas Untersuchung ist eine wichtige Darstellung einiger zentraler Konstruktionen des Udmurtischen und ihrer Verwendung. Sie ist freilich sehr theorielastig. Vielleicht hätte die quantitative Betrachtung des Materials einige Punkte weiter erhellt. Wenn es z. B. heißt, die Konstruktion N-Px. sei weiter verbreitet als in den anderen uralischen Sprachen (S. 69) oder die dependentmarking-Konstruktion werde seltener verwendet als in den anderen finnisch-ugrischen Sprachen, entsteht natürlich der Wunsch, exakte Kalkulationen zu sehen (S. 35). Die vorliegende Arbeit ist auch für die allgemeine Finnougristik von Bedeutung. Sie gibt Hinweise darauf, wie eines der zentralen Phänomene 
der Sprachfamilie, die Possessivsuffixe und insbesondere ihre Position beim Ausdruck der Possessivität und anderer Kategorien wissenschaftlich zu untersuchen sind.

\section{Esa-Jussi Salminen}

Alatyrev 1970 = Алатырев, В. И.: Выделительно-указательная категория в удмуртском языке. Ижевск: УдНИИ.

Heine, Bernd 1997: Possession: cognitive sources, forces and grammaticalization. Cambridge: Cambridge University Press.
Nikolaeva, Irina 2003: Possessive affixes in the pragmatic structuring of the utterance; evidence from Uralic. - Международный симпозиум по деиктическим системам и квантификации в языках Европь и северной и иентральной Азии. 130-145. Ижевск.

Suinkonen, Pirkko 2005: On the categories and functions developed from the possessive and deictic suffixes in Udmurt. - Lihkkun lehkos! Beiträge zur Finnougristik aus Anlass des sechzigsten Geburtstages von Hans-Hermann Bartens. 401-431. Hrsg. von C. Hasselblatt, E. KoPONEN, A. WidMER. Wiesbaden: Harrassowitz Verlag. 